\title{
Changes in resting connectivity with age: a simultaneous electroencephalogram and functional magnetic resonance imaging investigation.
}

\author{
Balsters JH, O'Connell RG, Galli A, Nolan H, Greco E, Kilcullen SM, Bokde AL, Lai R, Upton N, Robertson
}

$\mathrm{IH}$.

\begin{abstract}
Resting fluctuations in the blood oxygenation level-dependent signal have attracted considerable interest for their sensitivity to pathological brain processes. However, these analyses are susceptible to confound by nonneural physiological factors such as vasculature, breathing, and head movement which is a concern when investigating elderly or pathological groups. Here, we used simultaneous electroencephalogram (EEG) and functional magnetic resonance imaging (fMRI) (EEG/fMRI) to constrain the analysis of resting state networks (RSNs) and identify aging differences. Four of 26 RSNs showed fMRI and EEG/fMRI group differences; anterior default-mode network, left frontal-parietal network, bilateral middle frontal, and postcentral gyri. Seven RSNs showed only EEG/fMRI differences suggesting the combination of these 2 methods might be more sensitive to age-related neural changes than $\mathrm{fMRI}$ alone. Five RSNs showed only fMRI differences and might reflect nonneural group differences. Activity within some EEG/fMRI RSNs was better explained by neuropsychological measures (Mini Mental State Examination and Stroop) than age. These results support previous studies suggesting that age-related changes in specific RSNs are neural in origin, and show that changes in some RSNs relate better to elderly cognition than age.
\end{abstract}

Keywords: Aging; Simultaneous EEG/fMRI; Resting-state networks; Default mode network; Hippocampus; Prefrontal cortex

\section{Introduction}

Spontaneous, or resting state, fluctuations in the blood oxygenation level-dependent (BOLD) signal are providing useful insights into functional connectivity between brain regions, and how such connectivity is affected by various pathologies. A characteristic set of coactivating functional systems, typically referred to as resting state networks (RSNs), have been consistently identified in the brain across multiple studies (Allen et al., 2011; Biswal et al. 2010), and clinical populations (Fox and Greicius, 2010; Zhang and Raichle, 2010). These RSNs include basic sensory networks involved in visual, auditory, or sensorimotor processing, or more functionally complex networks such as the default-mode network (DMN) and frontal-parietal attention network (FPN). Numerous resting state studies have been conducted on aging populations and robust differences have been reported, particularly within the DMN (Buckner et al., 2008). However, the interpretation of RSN differences in aging and clinical research is complicated by the fact that the BOLD response is susceptible to nonneural factors including

\footnotetext{
* Corresponding author at: Trinity College Institute of Neuroscience, Trinity College Dublin, Dublin 2, Ireland. Tel.: +353 1896 8405; fax: +353-1-896 3183. E-mail address: balsterjotcd.ie (J.H. Balsters).
}

head movement, respiration, and vasculature. The present study explores the utility of electroencephalogram (EEG)-informed functional magnetic resonance imaging (fMRI) to isolate RSN differences that are truly neural in original.

One of the most robust and well-replicated findings in resting state studies of aging is that DMN connectivity decreases with age, an effect that has been observed at rest (Allen et al., 2011; Biswal et al., 2010; Damoiseaux et al., 2008; Lustig et al., 2003) and during task performance (Andrews-Hanna et al., 2007; Grady et al., 2010). Andrews-Hanna et al. (2007) showed that age-related decreases in DMN functional connectivity positively correlated with reduced structural integrity (decreased white matter [WM] integrity in the cingulum) and task performance (semantic memory task). Rather than focusing on the DMN, Damoiseaux et al. (2008) used independent component analysis (ICA) to investigate agerelated changes in 13 RSNs common to young and old participants including basic sensory regions and the DMN and FPN. Damoiseaux et al. (2008) found that only the DMN showed a significant aging effect, and unlike Andrews-Hanna et al. (2007) this ICA-based approach did not yield significant differences in the left or right FPN. DMN differences in Damoiseaux et al. (2008) withstood additional correction for gray matter (GM) volume, suggesting a functional rather than a structural deficit, and 
correlated with performance on the trail making test. A number of studies have shed light on the likely origins of DMN activity changes by demonstrating that the structural connections between nodes of the DMN decrease with age (Andrews-Hanna et al., 2007; Damoiseaux et al., 2009; Teipel et al., 2010).

The robust nature of these aging effects has been further underlined using large cohorts of resting state data to increase statistical power. Biswal et al. (2010) collected a sample of 1093 participants and demonstrated decreases in posterior regions of the DMN and left FPN with age. With a sample of 603 participants, Allen et al. (2011) showed that all 28 RSNs within their study were significantly modulated by age (all decreasing with age except for the basal ganglia and medial portion of the superior frontal gyrus which showed increases with age). Allen et al. (2011) also reported the first investigation of the spectral properties of RSN time courses in aging, and the correlations between RSNs. These additional RSN measures (BOLD spectral power and functional network connectivity [FNC]) were equally sensitive to aging because all 28 RSNs show a decrease in spectral power $(0.01-0.15 \mathrm{~Hz})$ with age, and connectivity between most RSNs decreased with age.

Though there is general consensus within the literature that connectivity in the DMN decreases with age, the results for other RSNs such as the FPN vary depending on the sample size and the analytical approach taken. Aside from the variability of results for certain RSNs another cause for concern is that RSNs might be susceptible to nonneural factors such as vasculature, breathing, or head movement (Cole et al., 2010; Iannetti and Wise, 2007; Kelly et al., 2012). For example, it is well established that age-related differences in vasculature lead to differences in the hemodynamic response function that can produce false positive results in fMRI studies (D'Esposito et al., 1999; Kannurpatti et al., 2010). It has also been demonstrated that variation in breathing can produce signal variations in regions with high blood volume such as GM (Birn et al., 2006; Wise et al., 2004). Although it has been repeatedly shown that connectivity in the DMN decreases with age, Birn et al. (2006) have also shown that the topography of voxels correlated with respiration variation-induced signal strongly resembles the DMN. Therefore age-related differences in respiration variation, and vasculature, could produce nonneural differences in RSNs. A third confounding variable is head movement. Two recent studies have suggested that differences in resting connectivity, particularly within the DMN and FPN, are partially because of head movement (Power et al., 2012; Van Dijk et al., 2012). Considering that head movement has been shown to differ in studies of aging (Allen et al., 2011: D'Esposito et al., 1999) it is important to ascertain whether differences in head movement are driving differences in RSNs.

All of these potential confounds can be addressed to some extent using multi-modal imaging, specifically the fusion of simultaneously recorded EEG and fMRI data. EEG provides a direct measure of postsynaptic neural activity and its combination with fMRI will further support the suggestion that differences in RSNs are neural in origin and not a by-product of vascular or breathing differences in populations. Unfortunately, EEG-informed fMRI is not immune to head movement contamination (Jansen et al., 2012; Moosmann et al., 2009) but steps can be taken to reduce the effect of this confound during the analysis (these procedures are discussed in detail in section 2. Methods).

For the first time we use simultaneous EEG and fMRI (EEG/fMRI) to investigate differences in RSNs that occur with healthy aging. We begin by analyzing EEG and fMRI data separately to replicate previous findings from the literature such as reduced alpha power with age (Babiloni et al., 2006a; Klimesch, 1999) and reduced DMN activity (Allen et al., 2011: Andrews-Hanna et al., 2007: Damoiseaux et al., 2008; Lustig et al., 2003). We then use EEG-informed fMRI to determine whether resting state differences are common to both modalities and thus likely to be neural in origin. Finally we correlate changes in RSNs validated using EEG-informed fMRI with changes in neuropsychological variables to better understand how aging effects specific cognitive domains through reduced functional connectivity within and between RSNs.

\section{Methods}

\subsection{Participants}

Fifteen young ( $18-28$ years old; mean age, $23.4 \pm 3.3$ years) and 27 elderly (65-78 years old; mean age, $71 \pm 4.49$ years) right handed, sex-matched participants were included in this study (see Table 1 for demographic details). Participants gave written informed consent before the study which was approved by the Trinity College Dublin School of Psychology Ethics Committee. On a day separate from the EEG/fMRI testing (elderly group: $163.43 \pm$ 118 days; young group: $161 \pm 81$ days), participants also underwent a neuropsychological battery consisting of the Mini Mental State Examination (MMSE; Folstein et al., 1975), the National Adult Reading Test (NART; estimate of intelligence; Nelson, 1982), the Stroop test, category fluency (animal), the Logical Memory subtest of the Wechsler Memory Scale III (WMS; Weschler, 1998), and the Hospital Anxiety and Depression Scale (Zigmond and Sims, 1983). Participants who scored more than 8 on either the anxiety or depression subscales of the Hospital Anxiety and Depression Scale were excluded from the study.

Participants were not taking any psychiatric or neurological medications at the time of testing.

\subsection{EEG/fMRI acquisition}

Participants lay supine in a magnetic resonance imaging (MRI) scanner (Philips 3T Achieva MRI Scanner, Trinity College Dublin) viewing visual stimuli in a mirror positioned above their face. Stimuli were presented using Presentation software v14.2 (Neurobehavioral Systems, Inc). EEG recordings were acquired with a 32-channel magnetic resonance-compatible BrainAmp system (Brainproducts, Munich, Germany). Thirty-three EEG electrodes were placed on the scalp, including the reference electrode positioned at FCz and the ground electrode placed at position AFz. One

Table 1

Participant demographic characteristics

\begin{tabular}{lcccc}
\hline Characteristic & Old $(n=27)$ & Young $(n=15)$ & \multicolumn{1}{l}{$t$} \\
\hline Age (y) & $71(0.86)$ & $23.40(0.86)$ & -35.86 & $<0.001$ \\
Sex & $15 / 27$ female & $4 / 15$ female & -1.83 & 0.075 \\
Neuropsychology & & & & \\
$\quad$ Nart (Z score) & $1.32(0.7)$ & $0.95(0.12)$ & -2.76 & 0.009 \\
$\quad$ Logical memory & $0.66(0.18)$ & $0.42(0.3)$ & -0.7 & 0.49 \\
$\quad$ (Z score) & & & & \\
MMSE & $28.3(0.21)$ & $29.18(0.26)$ & 2.43 & 0.02 \\
$\quad$ Animal fluency & $45.70(1.55)$ & $59.45(1.81)$ & 5.09 & $<0.001$ \\
$\quad$ Stroop (T score) & $21.33(0.94)$ & $25.73(2.69)$ & 1.96 & 0.058 \\
Structural & & & & \\
$\quad$ Gray matter (mL) & $576.96(9.09)$ & $664.84(10.19)$ & 6.1 & $<0.001$ \\
$\quad$ White matter (mL) & $519.95(10.09)$ & $539.52(10.31)$ & 1.25 & 0.217 \\
$\quad$ Cerebrospinal & $295.19(7.54)$ & $265.67(8.48)$ & -2.47 & 0.018 \\
$\quad$ fluid (mL) & & & & \\
$\quad$ Total intracranial & $1392.1(20.67)$ & $1470.04(20.87)$ & 2.36 & 0.023 \\
$\quad$ volume (mL) & & & & \\
Head movement & & & & \\
$\quad$ Translation (mm) & $0.087(0.0074)$ & $0.049(0.0043)$ & -3.61 & 0.001 \\
$\quad$ Rotation (radians) & $0.0005(0.00003)$ & $0.0004(0.00003)$ & -3.57 & 0.001 \\
\hline
\end{tabular}

Mean values reported, standard error in parentheses. Negative $t$ value indicates old $>$ young. Significant $p$ values are shown in bold.

Key: MMSE, Mini Mental State Examination. 
external electrode was applied to the subjects back to acquire the electrocardiogram (ECG). Electrode impedances were maintained at less than $10 \mathrm{k} \Omega$. The resolution and dynamic range of the EEG acquisition system were $100 \mathrm{nV}$ and $\pm 3.2 \mathrm{mV}$, respectively. Data were recorded on a laptop computer using Brain Recorder v1.04 software (BrainProducts, Munich, Germany) at a sampling rate of 5 $\mathrm{kHz}$ with a band-pass filter of $0.016-250 \mathrm{~Hz}$. Event timings and transistor-transistor logic (TTL) pulses from the MRI scanner at the onset of each volume acquisition were marked within the EEC trace. TTL pulses were also used to drive the visual stimuli in Presentation. The EEG clock was synchronized to the MRI scanner clock using the Brain Products Sync-box (Mullinger et al., 2008).

A high-resolution T1-weighted anatomic magnetizationprepared rapid gradient-echo image (field of view $=230 \mathrm{~mm}$, thickness $=0.9 \mathrm{~mm}$, voxel size $=0.9 \mathrm{~mm} \times 0.9 \mathrm{~mm} \times 0.9 \mathrm{~mm}$ ), and phase and magnitude maps were acquired first (echo time $1\left[\mathrm{TE}_{1}\right]=$ $1.46 \mathrm{~ms}, \mathrm{TE}_{2}=7 \mathrm{~ms}$ ). Each participant then underwent an echo planar imaging (EPI) session containing 210 volumes ( 7 minutes). The field of view covered the whole brain, $224 \mathrm{~mm} \times 224 \mathrm{~mm}$ ( $64 \times$ 64 voxels), 34 axial slices were acquired ( $0.05-\mathrm{mm}$ slice gap) with a voxel size of $3.5 \mathrm{~mm} \times 3.5 \mathrm{~mm} \times 4 \mathrm{~mm}$; repetition time [TR] =2 seconds, TE $=32 \mathrm{~ms}$, and flip angle $=78^{\circ}$. This was a sparsesampling sequence with the slices compressed to the first 1700 $\mathrm{ms}$ of the TR, leaving $300 \mathrm{~ms}$ uncontaminated by the magnetic resonance gradient artifact. This approach allowed us to visually inspect the relationship between TR markers recorded in the EEC trace and the onset of each EPI acquisition.

In the resting state task, participants kept their eyes open for 30 seconds (during this time the screen displayed "OPEN" in large font). After 30 seconds, the screen displayed "CLOSED" and participants closed their eyes. Participants then received a tap on the leg 30 seconds later to open their eyes again (again the screen displayed "OPEN"). This cycle was repeated 7 times, giving seven 30 second blocks of eyes open and seven 30-second blocks of eyes closed resting data lasting for 7 minutes in total. Visual instructions were used because in pilot studies it was determined that older participants could not hear an auditory tone because of the noise of the MRI scanner. This approach ensured that participants opened and closed their eyes as instructed. This resting state paradigm has the additional advantage of investigating 2 types of alpha activity: alpha reactivity (referred to as induced alpha in this report) and tonic alpha, which have been shown to correspond to separate neural networks (Ben-Simon et al., 2008).

\subsection{EEG preprocessing and analysis}

EEG data were preprocessed using a combination of in-house Matlab code, EEGLAB (Delorme and Makeig. 2004), and EEGLAB plugins (fmrib plugin [Niazy et al., 2005] and FASTER plugin [Nolan et al., 2010]). The optimal basis set approach developed by Niazy et al. (2005) was used to remove gradient artifacts. Data were then downsampled to $500 \mathrm{~Hz}$ and filtered between $1-30 \mathrm{~Hz}$ with an additional $50 \mathrm{~Hz}$ notch filter. QRS complexes were automatically detected in the ECG trace and 4 optimal basis functions of the balistocardiogram (BCG) template removed. ICA was run using the Infomax algorithm (Bell and Sejnowski, 1995) using the FASTER plugin (Nolan et al., 2010) which also automatically detected and removed artifactual independent components (ICs). Remaining bad channels were detected and interpolated. ICs were inspected visually and remaining eye movement artifacts were removed manually. Debener et al. (2008) suggest that template-based methods, including the optimal basis set approach, are not adequate for removing BCG artifacts and should be supplemented by removing additional BCG-ICs. However, it is also quite likely that with fewer than 64 channels, and noisy EEG data acquired during
fMRI, ICs are not completely unmixed and might contain elements of signal and artifact. This is more likely to be the case when running ICA over whole time courses as in resting state compared with event-related designs in which it is possible to run ICA around events of interest. To make sure that signal of interest was not removed we used a statistical rescaling function to rescale the remaining IC time courses at areas of high amplitude which are most likely noise (details in Supplementary data). Resting data were then epoched around TR markers ( $0-2000 \mathrm{~ms}$ ), baseline-corrected (0-100 ms), average referenced (returning the reference electrode FCz; TP9 and TP10 were not included when calculating the average reference because the signal quality of these electrodes was unreliable), and artifactual epochs were removed. Artifactual epochs were defined using the joint probability function in EEGlab (pop_jointprob.m) which assesses whether a single channel within an epoch deviates from the norm of that epoch, or whether the epoch as a whole deviates from the norm of all epochs. For singlechannel and whole epoch joint probability, deviations of greater than 3 standard deviations were rejected.

Eyes-closed and eyes-open resting EEG band power was calculated using the discrete Fourier transform. Absolute power values are sensitive to confounds of head volume conduction and so to counteract this problem we used relative power measures (Clarke et al., 2011; Klimesch, 1999; Moretti et al., 2004; Nuwer, 1988). Relative power measures were calculated by dividing the absolute power within the specific frequency band by absolute power for the range of interest $(1.5-30 \mathrm{~Hz})$. Relative power values were calculated separately for each frequency band (delta: $1.5-3.5 \mathrm{~Hz}$; theta: 4-7.5 $\mathrm{Hz}$; alpha 1: $8-11.5 \mathrm{~Hz}$; alpha 2: $12-13.5 \mathrm{~Hz}$; beta: $14-30 \mathrm{~Hz}$ ) across each region of interest (frontal [F3, F4, Fz], central [C3, C4, $\mathrm{Cz}]$, parietal [P3, P4, Pz], occipital [01, O2, Oz]). Two-way analyses of variance (ANOVAs) were run using factors of group ( 2 levels; young and old), and region (4 levels; frontal, central, parietal, occipital) across all 5 frequency bands (delta, theta, alpha 1, alpha 2, beta).

\section{4. fMRI preprocessing and analysis}

Scans were preprocessed using SPM8 (www.fil.ion.ucl.ac.uk/ spm). Before preprocessing. EPI data quality tests were conducted using the criterion defined in Iannetti et al. (2005). These include assessing the mean and standard deviation of the signal time course, image signal to noise, standard deviation of the single-voxel signal time course, and visual inspection for ghost artifact and signal dropout. All EPI data collected passed these tests. Images were then realigned and unwarped using field maps to correct for motion artifacts, susceptibility artifacts and motion by susceptibility interactions (Andersson et al., 2001; Hutton et al., 2002). This approach is superior to standard realignment procedures because it accounts for some of the nonlinear effects of head movement. Images were subsequently normalized to the International Consortium for Brain Mapping (ICBM) EPI template using the unified segmentation approach (Ashburner and Friston, 2005). Lastly, a Gaussian kernel of $8 \mathrm{~mm}$ Full Width Half Maximum (FWHM) was applied to spatially smooth the image. Slice-time correction was not performed as part of the preprocessing, because the relatively short TR ( 2 seconds), and the interleaved slice acquisition would sufficiently reduce the slice-time problem. GM, WM, cerebrospinal fluid (CSF), and total intracranial volume were calculated from structural images after segmentation using the VBM8 toolbox ( $\mathrm{r} 435$ ).

The preprocessed fMRI data were input into a single group spatial ICA as implemented in GIFT v2.0e (http://mialab.mrn.org/ software/gift/index.html). In this approach, principal component analysis was used to compress the data sets so that all subjects could be analyzed as a single group. Spatial ICA was then performed using the Infomax algorithm (Bell and Sejnowski, 1995), with 
subsequent back reconstruction into single subjects (Calhoun et al., 2001). The resulting output is an IC map and associated time course for every component and subject. Minimum descriptive length criteria ( $\mathrm{Li}$ et al., 2007) determined that the optimal number of independent components was 40 and ICASSO was run with 100 reruns and random initial conditions to ensure a robust decomposition (Himberg et al., 2004). Components with a quality $<0.9$ were excluded from further analysis. Components were also visually inspected for artifacts. This includes movement artifacts which present with a stereotypical 'ringing' around the edge of the cortex and activation maps which heavily overlapped with WM and CSF. Artifacts were inspected by 2 of the authors (JHB and ROC) and an additional colleague who was not an author on this report. All artifact inspectors reached consensus about which components to remove. Component labels were chosen using anatomical information and spatial multiple regression (comparing fMRI spatial maps to resting state networks found in Allen et al., 2011).

The Mancovan toolbox (Allen et al., 2011) was used to investigate the relationships between RSN measures (spatial map intensities [SMs]: BOLD spectral power; and FNC) and independent variables (age, GM, sex, neuropsychological measures, and head movement). Unfortunately, data entered into the Mancovan model must have a meaningful singular value per subject. This means it is not possible to include WM, CSF, and ECG information in the Mancovan model. However, these time courses were used in the EEG-informed fMRI analyses. Similar to standard fMRI analyses in which voxel beta weights specify the extent to which a voxel time course corresponds to a task time course, voxel intensity in a SM dictates the correspondence between a voxel time course and an IC time course (the greater the voxel intensity the greater the correspondence with the IC time course). Therefore, group differences in SMs reflect differences in the participation of a voxel, or cluster of voxels, in 1 of the RSNs. Spectral analyses are a common approach in EEG analyses, but a number of recent studies have shown that anatomical regions, and RSNs, have different BOLD spectral properties (Baria et al., 2011; Niazy et al., 2011; Salvador et al., 2008). FNC analysis allows one to investigate connectivity between RSNs rather than connectivity between voxel-based time courses. Though the ICs generated using this group ICA approach are spatially independent, it is still possible that significant temporal correlations exist between them. For example, Fox et al. (2005) previously demonstrated the strong anticorrelation between the DMN and FPN, and Kelly et al. (2008) showed that the degree of anticorrelation between these networks correlated with task performance. Previous studies have also shown that connectivity between RSNs differ with age (Allen et al., 2011) and schizophrenia (Jafri et al., 2008). FNC investigates the extent to which ICs temporally interact with each other and whether this connectivity between ICs differs across subjects. The details of this method are described in Jafri et al. (2008).

Multivariate analyses were first performed to assess the extent to which each of the independent variables explained variance in the data (Supplementary Figs. $1 \mathrm{~A}$ and $2 \mathrm{~A}$ ). At this stage redundant variables that do not explain significant variance in the data $(p>$ 0.05 ) are removed from the model. This procedure determines how well the independent variables explain variance within the dependent variables when other independent variables are taken into account. For example, Supplementary Fig. 1A (top panel) shows that activity within IC 11 is significantly modulated by age, however this activity modulation is better explained by head movement (specifically translation). Components will only be described as showing a significant age effect if age is the strongest predictor variable based on these multivariate analyses.

To determine which voxels, spectral bins, or connections between RSNs were correlated with age we additionally performed univariate analyses. Partial correlation was used to measure the strength of the linear relationship between 2 variables (e.g., log [power] and age) after adjusting for all other independent variables (head movement, sex, neuropsychological measures, etc.). Univariate tests were corrected for multiple comparisons at $p<0.05$ using false discovery rate (FDR; Genovese et al., 2002).

Considering the strong correlation between age and GM ( $r=-0.68 ; p=4.41^{-7}$ ), separate models were run with age orthogonalized with respect to GM, and GM orthogonalized with respect to age. Two linear regressions were used to calculate these residual values. Principal component analysis dimension reduction was performed on each model with the number of dimensions for spectra and FNC matrices specified using minimum descriptive length criteria. For the first 2 models (age and GM orthogonalized with respect to age or GM and age orthogonalized with respect to GM, sex, and head movement) spectra with 129 frequency bins were reduced to a range of 9 to 21 dimensions, and the FNC matrix with 325 pairwise correlations was reduced to 10 dimensions. Though spectra and FNC matrices have a Gaussian distribution, spatial maps often do not. This can make it difficult to reliably estimate the appropriate number of dimensions. Therefore all spatial maps (1699-12,766 voxels) were reduced to 13 dimensions. This value was chosen because it produced the lowest rate of false positive results and highest rate of true positive results in simulations run in Allen et al. (2011).

\subsection{EEG-informed fMRI}

EEG-informed fMRI refers to analyses that assess whether EEG fluctuations over time covary with fluctuations seen in the fMRI signal during the course of an experiment (see Huster et al., 2012, for a description of this and other EEG/fMRI analysis methods). In this study we assess whether the EEG power time courses are significant predictors of fMRI time courses after accounting for other regressors of no interest (head movement, ECG, CSF, and WM time courses). EEG power time courses were extracted using the same frequency boundaries described in section 2.3., on EEG preprocessing and analysis. Typically in EEG-informed fMRI studies, power time courses are generated by summing together all EEG electrodes (Balsters et al., 2011; Mantini et al., 2007). In addition to this, we also generated power time courses from specific electrode regions (frontal, central, parietal, and occipital EEG power time courses). In each case, EEG power time courses were downsampled to the TR sampling rate $(0.5 \mathrm{~Hz})$ and convolved with the informed basis set (Balsters et al., 2011; Friston et al., 1998). Epochs previously marked as bad (joint probability greater than $3 \mathrm{SDs}$ ) were replaced by the average of the one preceding and the one proceeding nonartifact data point as in other studies (Balsters et al., 2011; Eichele et al., 2005). Five general linear models (GLMs) were constructed for each EEG power time course region (i.e., frontal, central, parietal, occipital, and total). Each GLM consisted of the power time course of each frequency band (delta-beta), convolved with 3 functions of the informed basis set (canonical haemodynamic response function (HRF), temporal derivative, and dispersion derivative), and a number of regressors of no interest; individual head movement parameters generated during the realignment procedure, the principle eigenvariates from WM and CSF masks (masks were calculated from SPM WM and CSF templates in which the probability of being WM or CSF was greater than 50\%), and retrospective image correction (RETROICOR) using ECG time courses. RETROICOR regressors were generated using default settings within the PhLEM toolbox (https://sites.google.com/site/phlemtoolbox/) (Verstynen and Deshpande, 2011). An additional 5 GLMs were also generated in order to assess oscillatory changes unrelated to the transition between eyes-open or eyes-closed conditions (tonic EEG). These 
GLMs included all of the previously mentioned regressors, plus an additional condition describing the changes between eyes open and eyes closed ( 30 seconds on-off box car modeled as 3 regressors because of the convolution with the informed basis set). It is important to reiterate that all first-level GLMs contain EEG power time courses from each frequency band of interest (i.e., delta-beta) and regressors of no interest (head movement ( 3 translation and 3 rotation time courses), WM, CSF, and RETROICOR ECG time courses (sine and cosine components for the dominant Fourier series and their first harmonics). Separate GLMs only change the region from which the EEG data were extracted (i.e., frontal, central parietal, occipital or total). Temporal regression was used to compared fMRI IC time courses (dependent variable) with subject-specific GLMs (independent variables). Results were corrected for multiple comparisons (FDR-corrected, $p<0.05$ ).

\subsection{Mancovan on EEG-informed fMRI RSNs using neuropsychological measures}

A third Mancovan model was also run investigating the relationship between IC features and neuropsychological measures. Only RSNs that showed significant EEG-informed fMRI effects (group differences and frequency interactions) were included in this model. This model included age, NART, the Logical Memory subtest of the WMS, MMSE, animal fluency, and Stroop along with head movement response variables. In addition we included the time between neuropsychological testing and scanning as a regressor of no interest, along with an age by time interaction term in case the effect of the duration differed between groups. These regressors were removed from the Mancovan model in the backward selection process suggesting they did not explain a significant proportion of the variance. For this model, spectra were reduced to a range of 8 to 18 dimensions, and the FNC matrix was reduced to 9 dimensions. All spatial maps were reduced to 13 dimensions. Results were corrected for multiple comparisons (FDRcorrected, $p<0.05$ ).

\subsection{Anatomical localization}

Anatomical details of significant signal changes were obtained by superimposing the RSN images on the T1 canonical singlesubject image from the Montreal Neurological Institute series. The atlas of Duvernoy and Bourgouin (1999) was used as a general neuroanatomical reference. The SPM anatomy toolbox (Eickhoff et al., 2005) was used to establish cytoarchitectonic probabilities where applicable. The atlases of Diedrichsen et al. (2009) and Behrens et al. (2003) were employed as specific neuroanatomical references for cerebellar and thalamic activity, respectively. Terms such as 'prefrontal thalamus' refer to regions of the thalamus shown by Behrens et al. (2003) to be connected with the prefrontal cortex.

\section{Results}

\subsection{Neuropsychological differences}

Elderly participants performed significantly better on the NART $(t(36)=2.76 ; p<0.01)$ but significantly worse on the MMSE $(t(36)=2.43 ; p<0.05)$ and category (animal) fluency $(t(36)=5.09$; $p<0.001$ ). However, it should be noted that all participants had an MMSE score within the normal range $(>26)$. There were no significant differences in the Logical Memory subtest of the WMS III $(p=0.49)$ or Stroop scores $(p=0.058)$ between groups. Mean and standard error values are reported in Table 1.

\subsection{Age-related EEG oscillatory differences}

As in previous studies (Babiloni et al., 2006a, 2006b; Klimesch, 1999), 2-way ANOVAs showed significant decrease in alpha power with age in eyes-open (group by region interaction: alpha1: $F(3,105)=5 ; p<0.005$; alpha2: $F(3,105)=13.74 ; p<0.001)$ and eyes-closed conditions (group by region interaction: alpha1: $F(3,105)=3.03: p<0.05$; alpha2: $F(3,105)=9.51 ; p<0.001)$. These interactions were driven by a significant decrease in occipital alpha2 power with age (open: $t(41)=3.51 ; p<0.005$; closed: $\mathrm{t}(41)=4.19 ; p<0.001$ ). Significant group by region interactions were also found showing an increase in occipital theta with age (open: $F(3,105)=4.86 ; p<0.005)$; closed: $F(3,105)=4.28 ; p<$ 0.01 ). However, an analysis of peak alpha frequencies showed a slowing of oscillations across all regions with age (main effect of group: open: $F(1,35)=5.3 ; p<0.05$; closed: $F(1,35)=5.97 ; p<$ 0.05 ) with the greatest differences in occipital regions. We therefore suggest that the significant increase in occipital theta in the elderly group reflects the slowing of alpha frequency with age (Klimesch, 1999; Richard Clark et al., 2004). Fig. 1 shows EEG resting spectra and peak alpha frequency plots for each region and group.

\subsection{Age-related fMRI ICA differences}

After removing artifactual ICs based on decomposition quality (ICASSO; components with a quality $<0.9$; Himberg et al., 2004) and visual inspection, 26 out of 40 RSNs remained (all RSNs are displayed in Fig. 3). These RSNs were categorized as sensorimotor, visual, auditory, subcortical, attention, frontal, and DMN based on spatial overlap with RSNs presented in other studies (Allen et al., 2011; Cole et al., 2010; Damoiseaux et al., 2008). Details of activation clusters within each RSN are described in Supplementary Table 1. The Mancovan toolbox (Allen et al., 2011) was used to establish whether individual differences in RSN measures (SMs, spectra, or FNC) covaried with age and/or GM. EEG information was not used to constrain these initial analyses.

\subsection{Mancovan univariate analyses-age response variable}

Fig. 2A-C show significant univariate effects of age on RSN features; blue indicates a decrease in SM intensity/BOLD spectral power/connectivity with age and red indicates an increase in SM intensity/BOLD spectral power/connectivity with age. Fig. 2A shows increased SM intensity with age (red clusters) in the bilateral thalamus (prefrontal thalamic nuclei; IC 15), left parietal lobe (IC 26), and right precentral gyrus (area 6; IC 26). Decreased SM intensity with age (blue clusters) was observed in a separate portion of prefrontal thalamic nuclei (IC 14), right postcentral gyrus (areas 2 and 3b; IC 26), left inferior parietal lobule (IC 37), and the DMN (superior medial gyrus and posterior cingulate; IC 33). Fig. $2 B$ shows age effects on BOLD spectral power in the inferior frontal gyrus (IFG; IC 32) and prefrontal thalamus (IC 15). In both cases there was greater BOLD frequency power between 0 and $0.1 \mathrm{~Hz}$ for young participants. The prefrontal thalamic component (IC 15) also showed increased BOLD frequency power at a higher range $(0.15-0.25 \mathrm{~Hz})$ for elderly participants. Fig. 2C shows where connectivity between RSNs covaried with age. Elderly participants showed increased connectivity (FNC) between the prefrontal thalamus (IC 15) and both the posterior DMN (IC 19) and IFG (IC 34). However, in most cases connectivity between RSNs decreased with age. Young participants showed greater connectivity between a different prefrontal thalamic component (IC 14) and the same IFG component (IC 34) and right FPN (IC 40). There was also increased connectivity for young participants between motor (IC 39) and frontal components 
A Eyes open relative EEG spectra
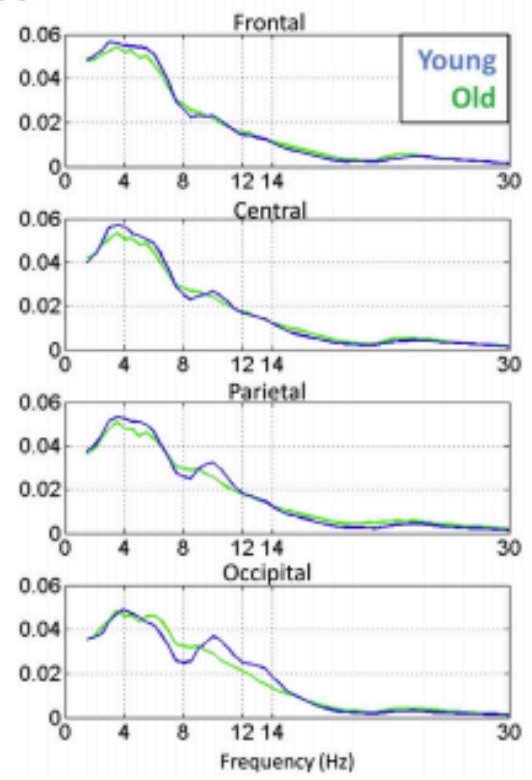

B

Open peak alpha frequency

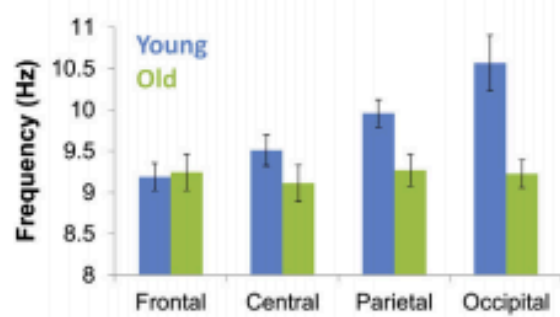

Eyes closed relative EEG spectra
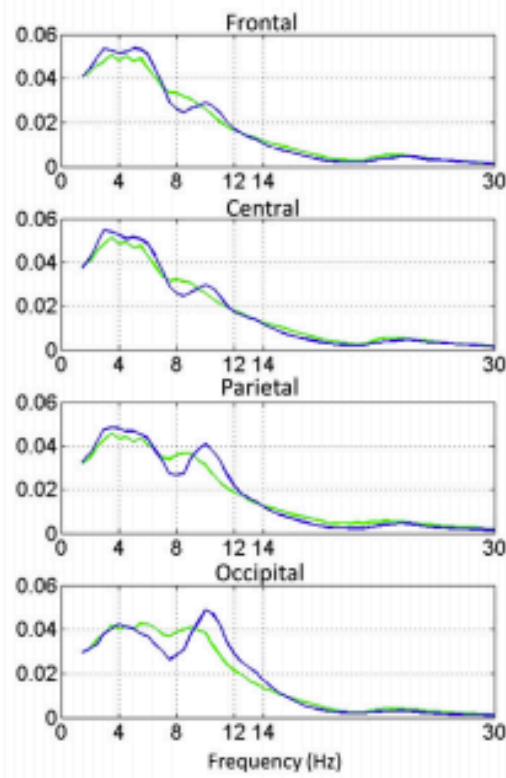

Closed peak alpha frequency

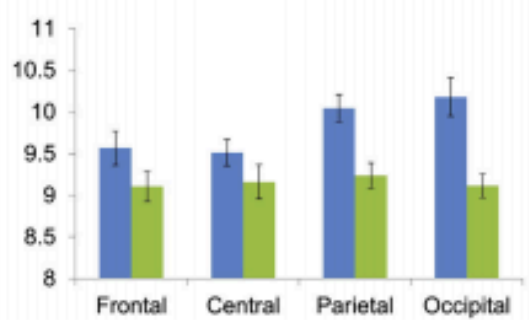

Fig. 1. EEG resting spectra: (A) relative power spectra are plotted for eyes open (left) and eyes-closed conditions (right). Dotted lines within each plot mark the boundaries of EEG frequency bands (delta, theta, alpha1, alpha2, beta). (B) Plots showing peak alpha frequency for young and old participants. Error bars represent standard error. In all graphs blue corresponds to the mean response of young participants, green corresponds to the mean response of old participants. Abbreviation: EEG, electroencephalogram.

(IC 36), medial premotor cortex (SMA; IC 30) and anterior DMN (IC 33) components, and attention (IC 28) and DMN (IC 1) components.

\subsection{Mancovan univariate analyses-GM response variable}

Fig. 2D-F show significant univariate effects of GM on RSN features; blue indicates a decrease in SM intensity/BOLD spectral power/connectivity with increased GM and red indicates an increase in SM intensity/BOLD spectral power/connectivity with increased GM. Fig. 2D shows that only the right postcentral gyrus (area 3b; IC 26) covaried with GM (increased signal intensity with increased GM). This region overlaps with a region in Fig. 2A where activity decreased with age. Fig. $2 \mathrm{E}$ shows that only the bilateral middle frontal gyrus (MFG; IC 36) increased in power $(0-0.1 \mathrm{~Hz})$ in concert with increasing GM. Fig. 2F shows that this same component showed a positive relationship between the amount of GM and connectivity with a DMN component (IC 1).

3.6. Mancovan univariate analyses-head movement and sex response variables

Head movement and sex were included as regressors of no interest in the fMRI only analyses. A number of components showed significant spatial map differences and spectral power differences related to head movement (Supplementary Figs. 3 and 4). These included ICs $4,10,11,12,13,15,19,25,26,30,32,34,35$, and 37 for SMs, and ICs 3, 4, 5, 10, 13, 19, 25, 26, 28, 29, 30, 32, 33, 34, 36, 37, 38 , 39, and 40 for spectral power. There was no significant effect of head movement on FNC. Although most components showed an effect of head movement it is important to reiterate that the multivariate analyses run on the data showed that age better explained the variance in the fMRI data than head movement (see Supplementary Figs. 1A and 2A). Also age effects presented in Fig. 2 have had all other modeled variables (including head movement) partialed out of the data thus these results cannot be explained by differences in head movement or any other of the modeled regressors. Sex was removed from the Mancovan model as part of the backward selection process suggesting that it did not explain any of the variance in the data.

\subsection{EEG-informed fMRI}

EEG-informed fMRI differences are reported in Table 2, and visualized in Fig. 3. We initially investigated EEG-informed fMRI differences using EEG total power time courses incorporating all electrodes. Using this approach we found frequency interactions in visual (ICs 4, 5), sensorimotor (ICs 3,17), frontal (IC 32), subcortical (IC 14), and DMN (IC 29). Significant frequency interactions refer to significant differences in the EEG-informed fMRI correlation across 


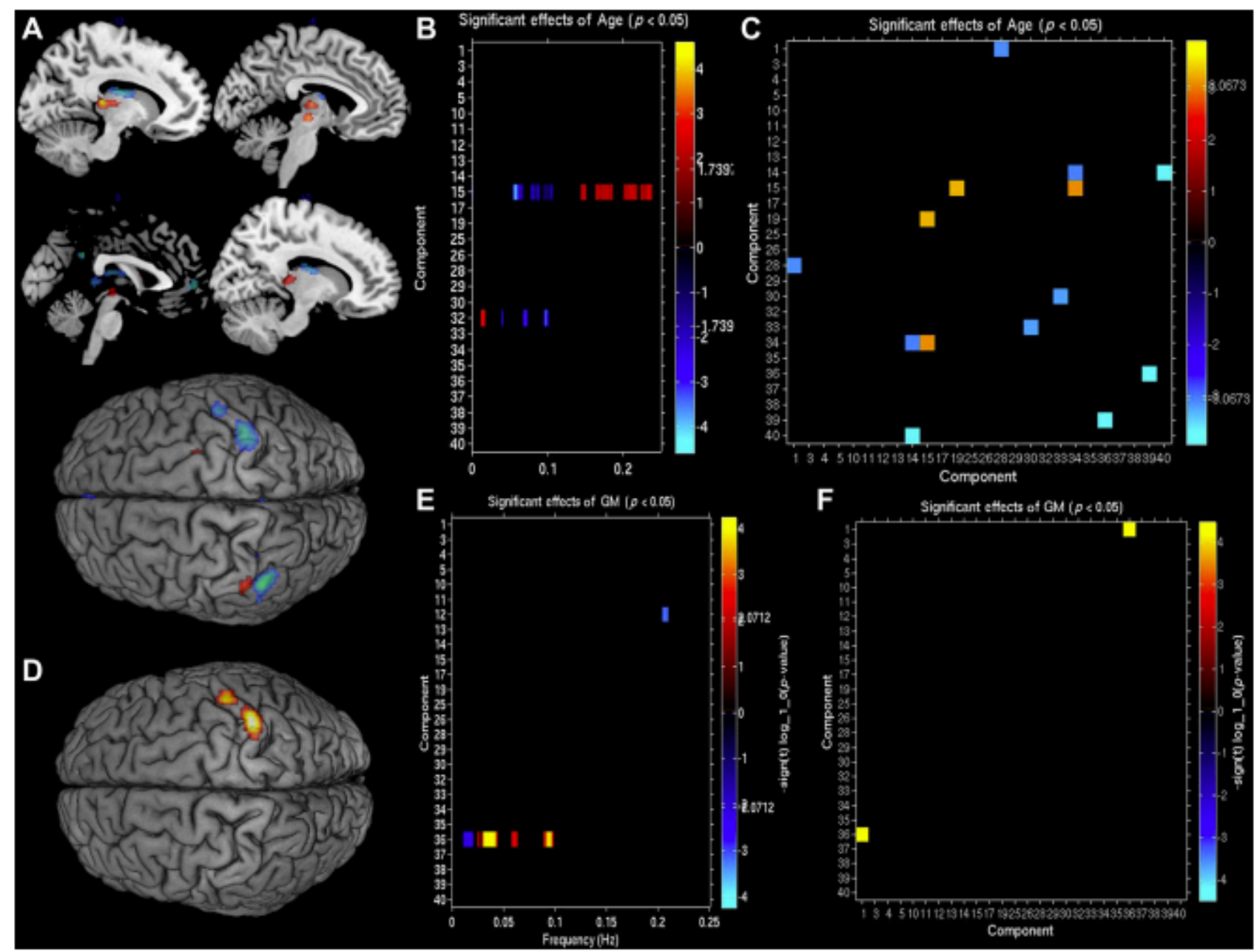

Fig. 2. Univariate test results showing the effects age and gray matter (GM) on IC measures: (A) and (D) show significant differences in spatial maps related to age and GM respectively. Activation maps are displayed in neurological orientation (left is left), false discovery rate-corrected ( $p<0.05$ ), and overlaid on a skull-stripped canonical brain. (B) and (E) Significant differences in spectral power related to age and GM respectively. (C) and (F) Significant differences in connectivity between RSNs because of age and GM respectively. In all figures blue represents a negative relationship between variables (increase activity/spectral power/connectivity with decreases in age/GM) and red/yellow indicates a positive relationship between RSN measures and variables (increase in activity/spectral power/connectivity with increases in age/GM). Abbreviations: IC, independent component; RSN resting state network.

modeled EEG frequency bands. For example, EEG-informed fMRI correlations in the visual ICs were significantly greater for the alpha1 frequency band compared with all other modeled EEG frequencies. Group by frequency interactions highlighted differences in visual (ICs 5, 10), SMA (IC 30), hippocampus (IC 35), left FPN (IC 37), and DMN components (IC 29).

Considering the group by region interactions found within specific frequency bands during the EEG analyses we also investigated EEG-informed fMRI differences using EEG power time courses derived from specific regions of electrodes (frontal, central, parietal and occipital electrodes). This approach highlighted additional frequency interactions in prefrontal thalamus (IC 15), posterior attention network (IC 28), and sensorimotor regions (IC 39), along with additional group by frequency interactions within visual (IC 13), sensorimotor (IC 26), anterior DMN (IC 33), and frontal RSNs (IC 36). It is clear from these results that though the spatial resolution of EEG is relatively limited, electrode selection is an important consideration when integrating EEG with fMRI. Supplementary Table 2 provides a breakdown of age-related EEGinformed fMRI differences for each frequency band. Fig. 3 shows RSNs that showed group differences in fMRI-only and EEG- informed fMRI analyses (purple activity) and RSNs that showed only EEG-informed fMRI group differences (blue activity).

\subsection{EEG-informed fMRI regressors of no interest}

The EEG-informed fMRI analyses included head movement, along with additional regressors of no interest; ECG, CSF, and WM time courses. Before correcting for WM, CSF, and ECG we found EEG-informed fMRI group differences in IC 36 (middle frontal gyri), IC 14 (thalamus), and IC 11 (basal ganglia), which were no longer significant when these regressors of no interest were included. However, adding these regressors of no interest highlighted EEGinformed fMRI group differences in IC 37 (left FPN), IC 13 (ventral occipital), IC 35 (hippocampus), and IC 26 (sensorimotor).

We also assessed whether group differences existed in our regressors of no interest which might overlap with our results of interest reported herein. As with the regressors of interest we ran ANOVAs on the beta values for the relationship between head movement and each IC time course. For simplicity we only report the results from the GLMs using EEG power time courses generated using all electrodes, however the results were similar across GLMs 


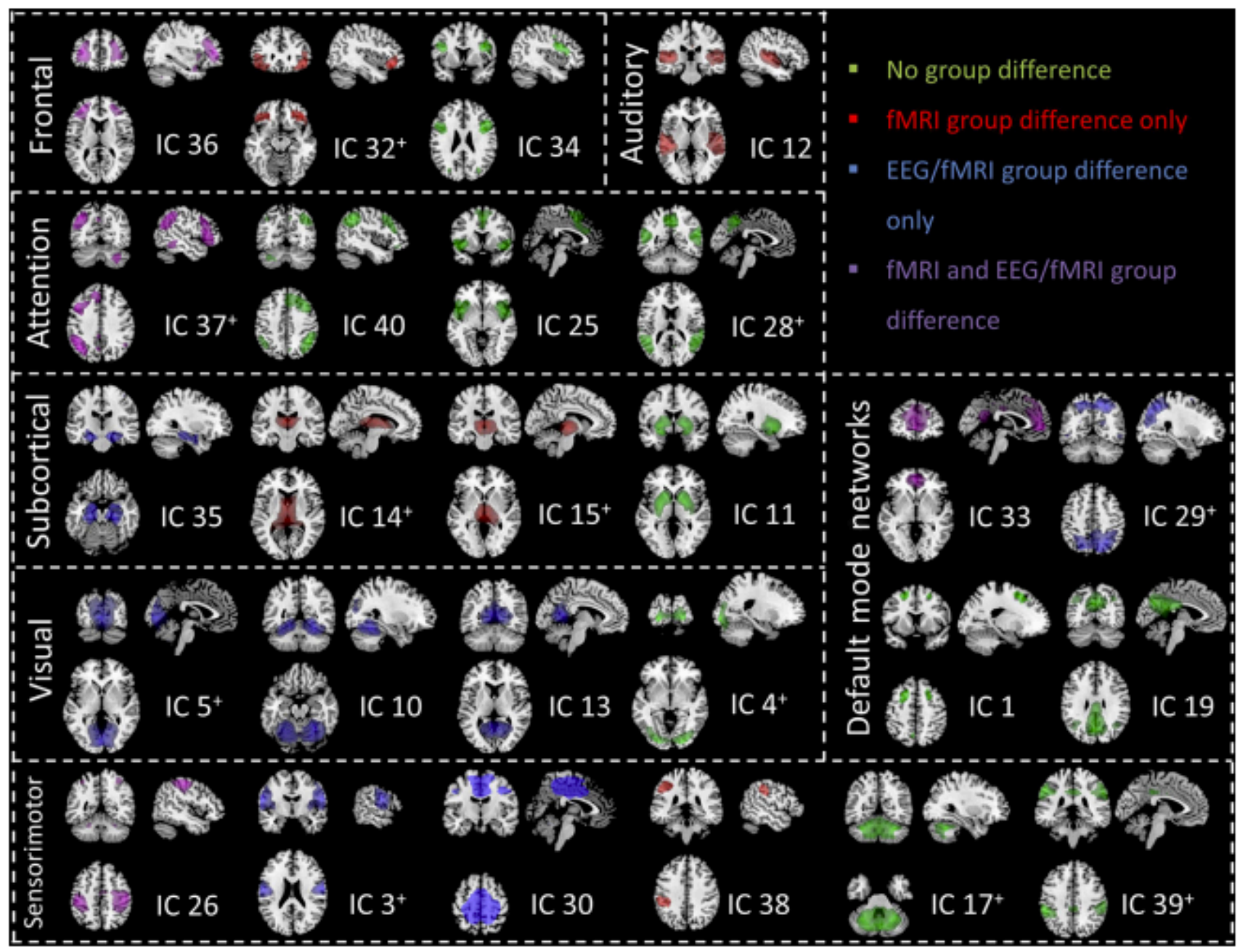

Fig. 3. Functional magnetic resonance imaging (fMRl) independent component analysis spatial maps: spatial $t$ maps are overlaid on a skull-stripped canonical brain thresholded at family-wise error $(p<0.05)$ with the component number underneath. Activation maps are displayed in neurological orientation (left is left). The color of the activation indicates whether there were fMRI-only group differences (red), electroencephalogram (EEG)-informed AMRI only group differences (blue), fMRI and EEG-informed fMRI group differences (purple), or no group differences (green). A cross next to the component number indicates there was an EEG-informed fMRI frequency interaction. Abbreviation: IC, independent component.

using EEG power time courses generated from other electrode sources. ICs $2,9,18,20$, and 28 all showed significant group differences related to head movement in a tonic GLM (only ICs 2,18 , and 28 showed significant group differences in head movement in the induced GLM). However, $4 / 5$ of these ICs were rejected as artifacts and as such not included in the previously reported fMRI-only model. There were no regions that showed a significant group difference in the relationship between functional networks and ECG.

\subsection{Neuropsychological correlates of EEG-informed fMRI RSNS}

Fig. 4 shows the results of a final Mancovan model run only on RSNs validated using EEG-informed fMRI. This model included age and head movement response variables as well as neuropsychological results (MMSE, NART, Logical Memory (LM), Stroop, and fluency). As in the previous analyses, age effects on SM intensity were found in the thalamus (IC 15) and postcentral gyrus (IC 26). Age effects on spectral power were found in the IFG (IC 32). These results were identical to those previously presented in Fig. 2. Considering the decrease in number of comparisons in the FNC analysis additional significant differences in connectivity were found in a number of extra RSNs. These included increased connectivity with age between the left FPN (IC 37) and thalamus (IC 15), and decreases in connectivity between (1) anterior DMN (IC 33) and posterior attention network (IC 28); (2) anterior DMN (IC 33) and SMA (IC 30); (3) hippocampus (IC 35) and visual cortex (IC 13); and (4) cerebellum (IC 17) and thalamus (IC 14).

NART, LM, and fluency did not show any relationship with RSN features. Stroop performance was positively correlated with increased activity within medial superior parietal lobe (area $5 \mathrm{M}$; IC 30), and increased spectral power within thalamic RSN (0.05-0.1 $\mathrm{Hz}$; IC 14). MMSE scores were negatively correlated with BOLD frequency power $(0.15-0.2 \mathrm{~Hz})$ within the bilateral MFG (IC 36). There were no differences in FNC related to neuropsychological measures. Multivariate analyses confirmed that these differences related to neuropsychological measures were not explained by age (see Supplementary Fig. 5A).

\section{Discussion}

Resting state fMRI studies have provided some of the most robust and well-replicated insights into age-related changes in brain function. However, an outstanding concern about this work has been the effect of nonneural differences such as vasculature, 
EEG-informed fMRI RSN effects

\begin{tabular}{|c|c|c|c|c|c|c|c|}
\hline $\begin{array}{l}\text { Component } \\
\text { number }\end{array}$ & Label & $\begin{array}{l}\text { Frequency } \\
\text { interaction }\end{array}$ & $\begin{array}{l}\text { Group by } \\
\text { frequency } \\
\text { interaction }\end{array}$ & Significant sensors and induced/tonic & $F$ value & $p$ value & Contributing frequency band \\
\hline 32 & Frontal & $\mathrm{Y}$ & & I_All & 2.104 & 0.01 & Alpha 2/beta \\
\hline 36 & Frontal & & $\mathrm{Y}$ & I_Cen & 2.684 & 0.000783 & Alpha $2 /$ beta \\
\hline 34 & Frontal & & & & & & \\
\hline 12 & Auditory & & & & & & \\
\hline 37 & Attention & $\mathrm{Y}$ & $\mathrm{Y}$ & LAll (G), T_All (G), L_Ooc (F), T_Occ (F) & $3.353 ; 2.593 ; 3.155 ; \mathbf{3 . 8 6 5}$ & $0.000032 ; 0.00119 ; 0.000084 ; 0.000002$ & Alpha 2 (F): alpha $2 /$ beta (G) \\
\hline 40 & Attention & & & & & & \\
\hline 25 & Attention & & & & & & \\
\hline 28 & Attention & $\mathrm{Y}$ & & 1_Par & 3.02 & 0.000161 & Alpha 2/beta \\
\hline 14 & Subcortical & $\mathrm{Y}$ & & LAll, T_All, I_Par, T_Par & $2.423 ; 2.472 ; 4.972 ; 3.965$ & $0.002576 ; 0.002069 ; 0 ; 0.000001$ & Alpha $2 /$ beta \\
\hline 11 & Subcortical & & & & & & \\
\hline 15 & Subcortical & $\mathrm{Y}$ & & T_Fron, T_Cen & $2.416 ; 2.584$ & $0.00265 ; 0.001245$ & Alpha 2/beta \\
\hline 35 & Subcortical & & $\mathrm{Y}$ & I_All, T_All & $3.479 ; 3.041$ & $0.000017 ; 0.000145$ & Beta \\
\hline 5 & Visual & $\mathrm{Y}$ & $\mathrm{Y}$ & LAll (F). T_All (G), T_Cen (G), L_Par (F), T_Par (F) & $\begin{array}{l}2.359 ; 4.898 ; 2.446 ; 3.97 ; \\
2.911\end{array}$ & $0.003427 ; 0 ; 0.002325 ; 0.000607 ; 0.000269$ & Alpha 1 \\
\hline 10 & Visual & & $\mathrm{Y}$ & I_All, T_All & $3.378 ; 2.977$ & $0.000028 ; 0.000197$ & Beta \\
\hline 4 & Visual & $\mathrm{Y}$ & & L_All, I_Fron, 1_Cen, L_Occ & $3.723 ; 6.27 ; 3.668 ; 2.952$ & $0.000005 ; 0 ; 0.000007 ; 0.00011 ; 0.000222$ & Alpha 1 \\
\hline 13 & Visual & & $\mathrm{Y}$ & T_Cen & 2.264 & 0.00519 & Alpha 2/beta \\
\hline 33 & DMN & & $\mathrm{Y}$ & I_Cen, T_Cen, L_Occ, T_Occ & $3.063 ; 3.458 ; 2.889 ; 2.881$ & $0.000131 ; 0.000019 ; 0.0003 ; 0.000312$ & Alpha 2/beta \\
\hline 29 & DMN & $\mathrm{Y}$ & $\mathrm{Y}$ & $\begin{array}{l}\text { 1.All (G/F), T_All (F), LFron (G/F), T_Fron (G/F), } \\
\text { T_Cen (F) }\end{array}$ & $\begin{array}{l}2.608(\mathrm{G}): 4.108(\mathrm{~F}): \\
4.18(\mathrm{~F}): 3.81(\mathrm{G}) ; 7(\mathrm{~F}): \\
3.778(\mathrm{G}): 5.216(\mathrm{~F}): \\
2.361(\mathrm{~F})\end{array}$ & $\begin{array}{l}0.001112(\mathrm{G}) ; 0.000001(\mathrm{~F}) ; 0 \text { (F); 3E-06 (G); } \\
\mathbf{0}(\mathrm{F}) ; 0.000004(\mathrm{G}) ; 0 \text { (F); } 000339(\mathrm{~F})\end{array}$ & Alpha 1 (F)/beta (G) \\
\hline 1 & DMN & & & & & & \\
\hline $\begin{array}{l}19 \\
3\end{array}$ & $\begin{array}{l}\text { DMN } \\
\text { Motor }\end{array}$ & $\mathrm{Y}$ & $\mathrm{Y}$ & LAll (F). I_Par (F), T_Par (F), L_Occ (G/F), T_Occ (G) & $\begin{array}{l}2.138 ; 3.139 ; 3.098 ; \\
2.258(\mathrm{G}) ; 2.643(\mathrm{~F}) ; 2.92\end{array}$ & $\begin{array}{l}0.0089 ; 0.00009 ; 0.00011 ; 0.005319(\mathrm{G}) ; \\
0.000946 \text { (F); } 0.000259\end{array}$ & Alpha 2, beta (F); alpha 1, delta (G) \\
\hline $\begin{array}{l}30 \\
38\end{array}$ & Motor & & Y & LAAll, I_Par, T_Par & $2.123 ; 3.517 ; 2.65$ & $0.009512 ; 0.000014 ; 0.000917$ & Alpha 2 \\
\hline 26 & $\begin{array}{l}\text { Motor } \\
\text { Motor }\end{array}$ & & $\mathrm{Y}$ & I Fron & 2.58 & 0.00126 & Theta/beta \\
\hline 17 & Motor & $\mathrm{Y}$ & & L_All, L_Fron, T_Fron, I_Cen, T_Cen, 1_Par, T_Par & $\begin{array}{l}2.248 ; 3.65 ; 3.886 ; 3.084 ; \\
2.989 ; 3.285 ; 3.559\end{array}$ & $\begin{array}{l}0.005557 ; 7 E-06 ; 0.000002 ; 0.000118 ; \\
0.000186 ; 0.000044 ; 0.000011\end{array}$ & Beta \\
\hline 39 & Motor & $\mathrm{Y}$ & & T_Fron & 2.209 & 0.006576 & Alpha $2 /$ beta \\
\hline
\end{tabular}

Summary of EEG-informed fMRI results. Significant frequency and group by frequency interactions are marked with a ' $\mathrm{Y}$ '. The electrode selection used to generate a significant result are reported in the next column (L, induced; $\mathrm{T}$ tonic; All, all electrodes; Fron, frontal electrodes [F3, F4, Fz]; Cen, central electrodes [C3, C4, CZ]; Par, parietal electrodes [P3, P4, Pz]; Occ, occipital electrodes [01, 02, Oz]]). (G) or (F) refers to whether it was a significant frequency interaction or group by frequency interaction. Values shown in bold indicate the largest effects where multiple results were significant. Component numbers correspond to components in Fig. 3.

Key: DMN, default mode network; EEG, electroencephalography; fMRI, functional magnetic resonance imaging; RSN, resting state network; $Y$, yes 


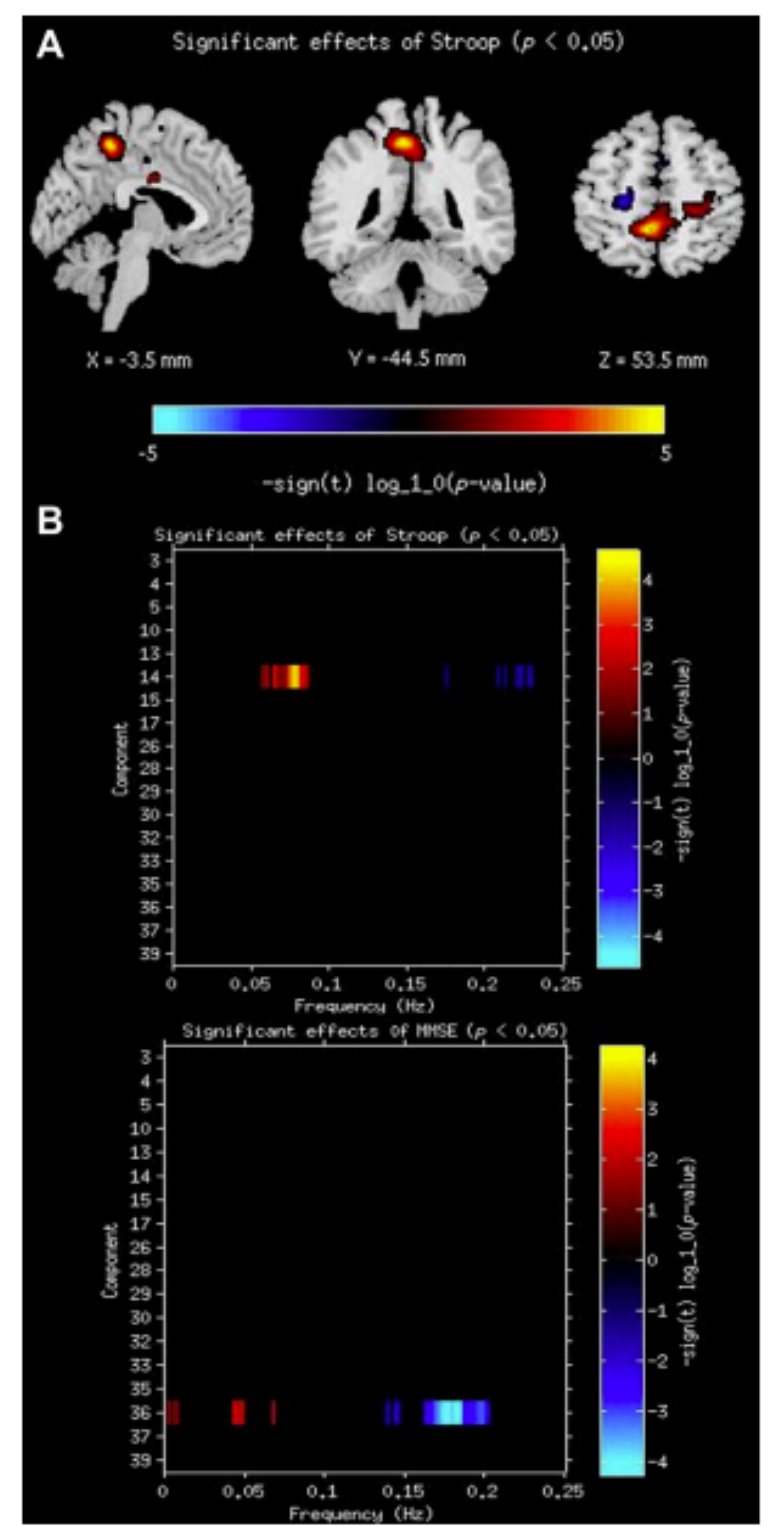

Fig. 4. Relationships between EEG-informed AMRI RSNs and neuropsychological variables: (A) Spatial map differences corresponding to Stroop performance. (B) Differences in BOLD spectra corresponding to Stroop and MMSE performance. In both (A) and (B) blue refers to a negative relationship (greater activation/spectral power, poorer performance), red to a positive relationship (greater activation/spectral power, better performance). Component numbers on the $Y$ axis of each plot correspond to component numbers in Fig. 3.

breathing, and head movement (all of which are likely to differ as a function of age) on resting connectivity (Cole et al., 2010; lannetti and Wise, 2007; Kelly et al., 2012). In the present study we sought to investigate this issue by using simultaneously acquired EEG, head movement, and ECG to constrain our fMRI analyses. Our EEGinformed fMRI analyses highlighted a number of RSNs showing significant age-related changes, including the DMN and left FPN. On the other hand, our study also highlights a number of RSN age effects in fMRI that were not supported by the inclusion of EEG data. It is possible that age effects seen in these RSNs are nonneural in origin. These results highlight the value of incorporating EEG data to improve detection of RSN effects.
The present study implemented a number of novel strategies to rule out potential nonneural confounds. The use of EEG to inform the fMRI analysis provided a means of excluding changes in vasculature and breathing on fMRI signal. Because EEG is susceptible to contamination by head movement artifacts (Jansen et al., 2012; Moosmann et al., 2009), our analyses included a number of additional steps: (1) field maps were used to unwarp fMRI data during realignment thus correcting for some nonlinear movement artifacts introduced by susceptibility by movement interactions (to our knowledge this has not been previously used in studies of resting state): (2) individual subject's movement time courses generated during the realignment stage were included in all GLMs as regressors of no interest; (3) all EEG frequency bands were modeled together in the same GLM removing movement signal common to all frequency bands and isolating the unique features of different frequency bands: (4) fMRI components that showed activity around the periphery of the cortex (stereotypical topography of movement artifacts) were removed; and (5) mean head movement values were additionally modeled in the Mancovan to address between-subject movement differences (within-subject head movement was additionally accounted for by steps 2 and 3). By combining all these safeguards, we can be confident EEGinformed fMRI group differences were not because of head movement but instead reflect connectivity differences in aging.

Our results show EEG-informed fMRI group differences (purple and blue activity in Fig. 3) in 11 of 26 RSNs, though 5 RSNs showed fMRI-only group differences (red activity in Fig. 3). It is possible that group differences within these RSNs are not neural in origin. Out of 11 RSNs showing EEG-informed fMRI group differences only 4 RSNs show group differences in fMRI-only and EEG-informed fMRI analyses; the anterior DMN (IC 33: age reduction in SM intensity). left FPN (IC 37: age reduction in SM intensity), bilateral postcentral gyrus (IC 26: age reduction in SM intensity), and MFG (IC 36: GM reduction in spectral power, 0-0.1 Hz). Seven RSNs showed EEGinformed fMRI group differences in the absence of fMRI differences (hippocampus [IC 35], posterior DMN [IC 29], visual [ICs 5, 10, and 13], and sensorimotor RSNs [ICs 3, 30]). In most cases group differences in fMRI activity were present within these RSNs at an uncorrected threshold (ICs 35, 29, 10, and 13), suggesting that EEGinformed fMRI may be more sensitive to neural changes that occur with age.

The DMN has been the most studied RSN, and appears to be a powerful biomarker for a number of clinical pathologies (Buckner et al., 2008), and healthy aging (Allen et al., 2011: Andrews-Hanna et al., 2007; Biswal et al., 2010; Damoiseaux et al., 2008). The DMN was also one of the first RSNs to be investigated using simultaneous EEG/fMRI and has been repeatedly found to correlate with beta band power time courses (Balsters et al., 2011; Laufs et al., 2003; Mantini et al., 2007). In this study we replicate the link between beta band power and DMN activity (IC 33), and show that differences in beta band power in young and old participants are correlated with decreases in DMN activity (Table 2). Decreases in DMN activity with age have been linked to poorer scores on tests of memory and executive functions (Andrews-Hanna et al., 2007; Damoiseaux et al., 2008). In addition to decreases in functional connectivity, Ystad et al. (2011) showed that decreased structural connectivity between the anterior DMN and subcortical structures (thalamus and putamen) were correlated with a proxy measure of executive function. In this study we used FNC to investigate the differences in connectivity between RSNs and showed age-related decrease in connectivity between the anterior DMN (IC 33) and the SMA (IC 30; both RSNs showed EEG-informed fMRI age-related differences). Connectivity between these 2 RSNs is achieved through the cingulum bundle (Schmahmann and Pandya, 2006). the same WM fiber bundle that connects the anterior and posterior 
nodes of the DMN (Greicius et al., 2009; Teipel et al., 2010). A number of diffusion tensor imaging (DTI) studies have shown that the integrity of the cingulum bundle decreases with age (AndrewsHanna et al., 2007; Damoiseaux et al., 2009; Michielse et al., 2010), leading to reduced functional connectivity between nodes of the DMN, and likely leading to reduced connectivity between the anterior DMN and SMA in this study. Unfortunately, the integrity of this WM tract has not been linked to a specific cognitive or neuropsychological metric. There is now a large body of evidence suggesting that the DMN decreases in functional and structural connectivity with age, and the present study further supports this. Future research is now required to elaborate on the cognitive consequences associated with the decrease in DMN activity which occurs with healthy aging.

Along with the DMN, hippocampal integrity is thought to be an important marker of healthy aging and dementia (Teipel et al. 2007). Considering that studies of rest and task have shown a strong coupling between the hippocampal formation and DMN fluctuations (Huijbers et al., 2011; Vincent et al., 2006) it is not surprising that in this study we found the DMN (IC 33) and hippocampus (IC 35) show age-related changes in function. For both the DMN and hippocampus it was the contribution of beta power (Table 2) that changed with age. However, our results show a decrease in beta DMN activity compared with an increase in beta hippocampal activity with age. This partially overlaps with the results of Andrews-Hanna et al. (2007) who showed a decrease in BOLD variability within the DMN with age but saw an increase in BOLD variability within the hippocampus with age. Although the hippocampus has been shown to decrease in volume with age (Fjell et al., 2009: Raz et al., 2005) a number of studies have shown increased hippocampal activity and frontal-hippocampal connectivity with age. For example, Daselaar et al. (2006) showed a selective age-related decrease in the hippocampus related to recognition, but an age-related increase within the rhinal cortex related to familiarity, along with greater frontal-rhinal connectivity in elderly participants. Similar results were found by O'Connell et al. (2012) using an attention paradigm (3 stimulus oddball). O'Connell et al. (2012) used EEG-informed fMRI to investigate age-related changes in the P3a (distractor related eventrelated potential [ERP] component) and P3b (target detection ERP component) and showed increased EEG-informed fMRI activation for elderly participants in the right hippocampus related to P3a and P3b components, and increased prefrontal cortical activity.

The EEG-informed fMRI analysis revealed a significant decrease in the left FPN (IC 37) in the elderly group. Resting state studies have yielded somewhat inconsistent findings regarding the extent to which prefrontal cortex activity changes with age. Damoiseaux et al. (2008) failed to show any differences in the left or right FPN with age, whereas Allen et al. (2011) showed significant aging decreases in BOLD spectra within all frontal RSNs and left and right FPN. Although left and right FPN showed significant decreases in SM intensity in Allen et al. (2011) and Biswal et al. (2010), significant decreases in these networks were driven only by decreased parietal activity and not decreased prefrontal activity. Our results replicate this finding, showing significant reduction in SM intensity for the left FPN, but only over the parietal cortex (see Fig. 2A). As in previous studies (Balsters et al., 2011; Mantini et al., 2007; Sadaghiani et al., 2010), EEG-informed fMRI analyses showed that this decreased FPN activity was correlated with the age-related decrease in alpha2 power (Table 2).

There is still a great deal of debate regarding the interpretation of prefrontal activity changes with age (see Grady, 2012 for a comprehensive review). Meta-analyses by Spreng et al. (2010) and Rajah and D'Esposito (2005) reported reliable prefrontal activity increases with age during task performance but a large scale longitudinal study by Nyberg et al. (2010) suggested that increasing cortical activity with age is an artifact of cross-sectional designs, and that longitudinal assessments show a significant decrease in prefrontal activity with age. It is not yet clear if the ability to upregulate activity within cognitive control regions, such as the FPN, is beneficial in aging. Davis et al. (2008) suggest that increased prefrontal activity is associated with improved elderly cognition, specifically the ability for elderly individuals to perform on par with young participants. Campbell et al. (2012) also showed that reduced FPN connectivity in elderly participants was correlated with greater distractibility during a 1-back task with multiple stimuli, suggesting that increased FPN connectivity has a positive effect on task performance in aging. However, studies by Grady et al. (2010) and Salami et al. (2012) found that increased FPN activity during a variety of tasks correlated with poorer performance. In this study we did not find that FPN activity correlated with any neuropsychological measurements so it is not clear the extent to which these resting differences in cognitive control regions correlate with subsequent changes in task performance. However, in a similar previous study Balsters et al. (2011) demonstrated a decrease in right FPN 6 hours after healthy elderly participants consumed $5 \mathrm{mg}$ of donepezil (acetylcholinesterase inhibitor). This decrease in right FPN activity, in the same resting paradigm used in this study, was associated with decreasing alpha2 power and positively correlated with decreases in performance on a paired associates learning task (similar to the task performed in Salami et al., 2012). It is therefore likely that a decrease in alpha-driven FPN activity with age is linked to reduced cognitive control and poorer performance.

Individual differences in resting fluctuations within the medial sensorimotor RSN (IC 30), thalamic RSN (IC 14), and middle frontal gyri (IC 36) (all RSNs validated using EEG-informed fMRI) correlated with neuropsychological measurements, specifically the Stroop and MMSE. Though a number of fMRI studies of the Stroop task have shown activity within the precuneus (Langenecker et al., 2004; Milham et al., 2002; Zysset et al., 2007), there is mixed evidence for an age-related difference in this region. Milham et al. (2002) found this region was more active for young compared with old participants, and Langenecker et al. (2004) showed this region was more active in old compared with young participants, and Zysset et al. (2007) found no aging difference in the precuneus. None of these studies reported thalamus activity during Stroop performance. However, these studies focused on aging differences, or subclassified age groups into high and low performers, whereas activity in this study correlated solely with Stroop performance. Multivariate analyses (Supplementary Fig. 5) suggest that activity within precuneus SMs and spectral power in the thalamus are better explained by Stroop performance than age. We therefore suggest that the precuneus activity and thalamic spectral power are important for inhibitory control regardless of age. Similarly, spectral activity within the MFG $(0.15-0.2 \mathrm{~Hz})$ was specifically predicted by MMSE performance and not by age (although BOLD spectral differences in this RSN were best explained by GM rather than age; see Supplementary Figs. $1 \mathrm{~A}$ and $2 \mathrm{~A}$ ). Multivariate tests also showed that head movement was a slightly more significant predictor of spectral activity than MMSE score (MMSE $p=0.013$; translation $p=$ 0.0094; this was not the case for either of the Stroop results; see Supplementary Fig. 5A) so these results must be interpreted with caution, although previous studies have also shown that resting activity within the MFG is negatively correlated with MMSE score (He et al., 2007).

As predicted, some RSNs that showed age-related changes in fMRI failed to show EEG-informed fMRI group differences. These included the auditory cortex (IC 12), both thalamic RSNs (ICs 14,15), sensorimotor RSNs (IC 38), and the IFG (IC 32). Although these RSNs failed to show an EEG/fMRI group difference it does not necessarily 
mean aging differences in these RSNs are nonneural in origin. At an uncorrected threshold the IFG (IC 32) and the dorsal medial nucleus of the thalamus (IC 15) show EEG-informed fMRI age effects. None of these components showed any significant group differences related to regressors of no interest in the EEG-informed fMRI analyses. Considering that the Mancovan toolbox is a between subjects analysis tool we were not able to use WM, CSF, or ECG information in the fMRI-only analyses because these values could not be transformed into a meaningful singular value. Unfortunately, respiration data were not collected in this study but respiration variation signals could explain age differences in other RSNs. It is also possible that group differences in these RSNs are not artifact but driven by oscillations in the gamma band. Gamma band EEG power has been shown to contribute to the BOLD response in a unique manner compared with alpha or beta oscillatory power (Scheeringa et al., 2011). However, the fMRI acquisition parameters used in our study were not ideal for reliably investigating gamma band power fluctuations. Future studies might acquire resting state data using a sparse sampling acquisition with a larger quiet period (perhaps 2 seconds compared with $300 \mathrm{~ms}$ used in this study) in which gamma band signal could be acquired without the contamination of gradient artifact.

Throughout this report, we have referred to ICs as 'resting state networks (RSNs)' to make them comparable with the existing literature, however this was not a standard resting state study. Rather than being told to relax, participants were instructed to open and close their eyes every 30 seconds with the aim of driving variability in alpha power across the time course (Ben-Simon et al., 2008; Berger, 1929). Although this introduces a task (participants were explicitly instructed when to open and close their eyes), there are several reasons to suggest our results are entirely comparable with those of standard resting state studies. First, most EEGinformed fMRI age differences were present for tonic and induced analyses (see Table 2), suggesting that age-related differences were not task-related. This was not the case for all RSNs; age differences within the MFG (IC 36), and postcentral gyrus (IC 26) were only significant for induced time courses and were not present when the task time course was removed. Second, it has been repeatedly shown that RSNs are not only present during rest but can also be seen during tasks (Grady et al., 2010; Smith et al., 2009). It is therefore likely that these 'resting state' networks reflect functionally linked systems that are not restricted to periods of rest.

In conclusion, we have shown using EEG/fMRI that the agerelated reduction in activity within specific RSNs is likely to be neural in origin. These networks include frontal, visual, sensorimotor, hippocampal, the left FPN, and DMN. We also show that activity within some of these RSNs is better explained by elderly cognition rather than age. Finally, we show that EEG/fMRI highlights group differences that were not present using fMRI alone, suggesting that combing techniques in this manner might be a more sensitive approach to developing biomarkers of aging.

\section{Disclosure statement}

The authors report no conflicts of interest.

Participants gave written informed consent before the study, which was approved by the Trinity College Dublin School of Psychology Ethics Committee.

\section{Acknowledgements}

This work was supported by funding from the GlaxoSmithKline/ Trinity College Institute of Neuroscience Research Consortium on Neurodegeneration. J.H. Balsters was supported by a fellowship from the Irish Research Council for Science Engineering and
Technology (IRCSET). A.L.W. Bokde was supported by Science Foundation Ireland Stokes Programme. Participant recruitment and assessment was supported by Atlantic Philanthropies Neuroenhancement for Independent Lives (NEIL) grant to L.H. Robertson.

\section{Appendix A. Supplementary data}

Supplementary data associated with this article can be found, in the online version, at http://dx.doi.org/10.1016/j.neurobiolaging. 2013.03.004

\section{References}

Allen, E.A, Erhardt EB, Damaraju, E Gruner, W, Segall, JM Silva, R.F. Havlicek, M., Rachakonda, S., Fries, J., Kalyanam, R., Michael, A.M., Caprihan, A. Turner, JA. Eichele, T., Adelsheim, S., Bryan, A.D., Bustillo, J., Clark, V.P., Feldstein Ewing. S.W., Filbey. F., Ford, CC., Hutchison, K., Jung. R.E. Kiehl, K.A. Kodituwakku, P. Komesu, Y.M. Mayer, A.R., Pearlson, G.D., Phillips, J.P., Sadek, J.R. Stevens, M., Teuscher, U., Thoma, R.J., Calhoun, V.D., 2011. A baseline for the multivariate comparison of resting-state networks. Front. Syst. Neurosci. 5. 2.

Andersson, J.L., Hutton, C., Ashburner, J., Turner, R., Friston, K., 2001. Modeling geometric deformations in EPI time series. Neuroimage 13, 903-919.

Andrews-Hanna, J.R., Snyder, AZ. Vincent, J.L, Lustig. C., Head, D., Raichle, M.E. Buckner, R.L., 2007. Disruption of large-scale brain systems in advanced aging Neuron 56, 924-935.

Ashburner, J., Friston, K.J., 2005. Unified segmentation. Neuroimage 26, 839-851. Babiloni, C., Binetti, G. Cassarino, A., Dal Forno, G., Del Percio, C. Ferreri, F., Ferri, R. Frisoni, G., Galderisi, S., Hirata, K., Lanuzza, B., Miniussi, C. Mucci, A., Nobili, F., Rodriguez, G., Luca Romani, G., Rossini, P.M., 2006a. Sources of cortical rhythms in adults during physiological aging: a multicentric EEG study. Hum. Brain Mapp. 27, 162-172.

Babiloni, C., Binetti, G., Cassetta, E. Dal Forno, G., Del Percio, C., Ferreri, F., Ferri, R. Frisoni, G., Hirata, K., Lanuzza, B., Miniussi, C. Moretti, D.V., Nobili, F. Rodriguez, G., Romani, G.L., Salinari, S., Rossini, P.M., 2006b. Sources of cortical rhythms change as a function of cognitive impairment in pathological aging: a multicenter study. Clin. Neurophysiol. 117, 252-268.

Balsters, J.H., O'Connell, R.G., Martin, M.P., Galli, A., Cassidy. S.M., Kilcullen, S.M. Delmonte, S., Brennan, S., Meaney, J.F., Fagan, A.J., Bokde, A.L., Upton, N., Lai, R., Laruelle, M. Lawlor, B., Robertson, L.H., 2011. Donepezil impairs memory in healthy older subjects: behavioural, EEG and simultaneous EEG/fMRI biomarkers. Pl.oS One 6, e24126.

Baria, A.T., Baliki, M.N., Parrish, T., Apkarian, A.V., 2011. Anatomical and functional assemblies of brain BOLD oscillations. J. Neurosci. 31, 7910-7919.

Behrens, T.E., Johansen-Berg, H., Woolrich, M.W., Smith, S.M., WheelerKingshott, CA., Boulby, PA., Barker, G.J., Sillery, E.I., Sheehan, K., Ciccarelli, O.
Ka., Thompson, A.J., Brady, J.M., Matthews, P.M., 2003. Non-invasive mapping of connections between human thalamus and cortex using diffusion imaging. Nat. Neurosci. 6, 750-757.

Bell, A.J., Sejnowski, T.J., 1995. An information-maximization approach to blind separation and blind deconvolution. Neural Comput. 7, 1129-1159.

Ben-Simon, E. Podlipsky, L., Arieli, A., Zhdanov, A., Hendler, T., 2008. Never resting brain: simultaneous representation of two alpha related processes in humans. PLoS One 3, e3984.

Berger, H., 1929. "About the electroencephalogragram in man". Eur. Arch. Psychiatry Clin. Neurosci. 87, 527-570.

Birn, R.M., Diamond, J.B., Smith, M.A., Bandettini, P.A., 2006. Separating respiratoryvariation-related fluctuations from neuronal-activity-related fluctuations in fMRI. Neuroimage 31, 1536-1548.

Biswal, B.B., Mennes, M., Zuo, X.N., Gohel, S., Kelly, C., Smith, S.M., Beckmann, C.F. Adelstein, J.S., Buckner, R.L., Colcombe, S., Dogonowski, A.M., Ernst, M., Fair, D., Hampson, M., Hoptman, M.J. Hyde, J.S, Kiviniemi, V.J.. Kotter, R., Li, S.J., Lin, C.P. Lowe, M.J., Mackay, C., Madden, D.J. Madsen, K.H., Margulies, D.S., Mayberg, H.S., McMahon, K., Monk, C.S., Mostofsky, S.H., Nagel, B.J., Pekar, J.J. Peltier, S.J. Petersen, S.E., Riedl, V., Rombouts, S.A., Rypma, B., Schlaggar, B. Schmidt, S., Seidler, R.D., Siegle, G.J., Sorg. C., Teng, G.J. Veijola, J., Villringer, A. Walter, M., Wang, L. Weng. X.C. Whitfield-Gabrieli, S., Williamson, P. Walter, M., Wang. L. Weng. X.C. Whitfield-Gabrieli, S., Williamson, P., Windischberger, C., Zang. Y.F., Zhang, H.Y., Castellanos, F.X., Milham, M.P., 2010. Toward discovery science of human brain function. Proc. Natl. Acad. Sci. U.SA
$107,4734-4739$.

Buckner, R.L., Andrews-Hanna, J.R., Schacter, D.L., 2008. The brain's default network anatomy, function, and relevance to disease. Ann. N. Y. Acad. Sci. 1124, 1-38.

Calhoun, V.D., Adali., T., Pearlson, G.D., Pekar, J.J.. 2001. A method for making group inferences from functional MRI data using independent component analysis. Hum. Brain Mapp. 14, 140-151

Campbell, K.L., Grady, C.L., Ng. C., Hasher, L., 2012. Age differences in the frontoparietal cognitive control network: implications for distractibility. Neuropsychologia 50, 2212-2223.

Clarke, A.R., Barry, R.J., Dupuy, F.E., Heckel, LD., McCarthy, R., Selikowitz, M., Johnstone, S.J. 2011. Behavioural differences between EEG-defined subgroups of 
children with Attention-Deficit/Hyperactivity Disorder. Clin. Neurophysiol. 122, 1333-1341.

Cole, D.M. Smith, S.M., Beckmann, CF., 2010. Advances and pitfalls in the analysis and interpretation of resting-state FMRI data. Front. Syst. Neurosci. 4, 8.

Damoiseaux, J.S., Beckmann, C.F., Arigita, E.J., Barkhof, F., Scheltens, P., Stam, C.J., Smith, S.M., Rombouts, S.A., 2008. Reduced resting-state brain activity in the "default network" in normal aging. Cereb. Cortex 18, 1856-1864.

Damoiseaux, J.S., Smith, S.M., Witter, M.P., Sanz-Arigita, E.J., Barkhof, F., Scheltens, P., Stam, C.., Zarei, M., Rombouts, SA., 2009. White matter tract integrity in aging and Alzheimer's disease. Hum. Brain Mapp. 30, 1051-1059.

Daselaar, S.M., Fleck, M.S., Dobbins, I.G., Madden, D.J. Cabeza, R., 2006. Effects of healthy aging on hippocampal and rhinal memory functions: an event-related fMRI study. Cereb. Cortex 16, 1771-1782.

Davis, S.W., Dennis, N.A., Daselaar, S.M., Fleck, M.S., Cabeza, R., 2008. Que PASA? The posterior-anterior shift in aging. Cereb. Cortex 18, 1201-1209.

Debener, S., Mullinger, K... Niazy, R.K., Bowtell, R.W., 2008. Properties of the ballistocardiogram artefact as revealed by EEG recordings at 1.5, 3 and $7 \mathrm{~T}$ static magnetic field strength. Int. J. Psychophysiol. 67, 189-199.

Delorme, A. Makeig, S., 2004. EECLAB: an open source toolbox for analysis of single-trial EEG dynamics including independent component analysis. J. Neurosci. Methods 134, 9-21.

D'Esposito, M., Zarahn, E, Aguirre, G.K., Rypma, B., 1999. The effect of normal aging on the coupling of neural activity to the bold hemodynamic response. Neuroimage 10, 6-14.

Diedrichsen, J. Balsters, J.H., Flavell, J., Cussans, E., Ramnani, N., 2009. A probabilistic MR atlas of the human cerebellum. Neuroimage 46, 39-46.

Duvernoy. H.M., Bourgouin, P., 1999. The Human Brain: Surface, Three-Dimensional Sectional Anatomy and MRI. Springer-Verlag. Wein, New York.

Eichele, T., Specht, K., Moosmann, M. Jongsma, M.L., Quiroga, R.Q., Nordby, H., Hugdahl, K. 2005. Assessing the spatiotemporal evolution of neuronal activation with single-trial event-related potentials and functional MRI. Proc. Natl. Acad. Sci. U.S.A 102, 17798-17803.

Eickhoff, S.B., Stephan, K.E., Mohlberg, H., Grefkes, C., Fink, G.R., Amunts, K., Zilles, K., 2005. A new SPM toolbox for combining probabilistic cytoarchitectonic maps and functional imaging data. Neuroimage 25, 1325-1335.

Fjell, A.M., Walhovd, K.B., Fennema-Notestine, C., McEvoy, L.K., Hagler, D.J. Holland, D., Brewer, J.B., Dale, A.M., 2009. One-year brain atrophy evident in healthy aging. J. Neurosci. 29, 15223-15231.

Folstein, M.F., Folstein, S.E., McHugh, P.R., 1975. "Mini-mental state". A practical method for grading the cognitive state of patients for the clinician. J. Psychiatr. Res. 12, 189-198.

Fox, M.D., Greicius, M., 2010. Clinical applications of resting state functional connectivity. Front. Syst. Neurosci. 4, 19.

Fox, M.D., Snyder, AZ. Vincent, J.L., Corbetta, M. Van Essen, D.C. Raichle, M.E., 2005. The human brain is intrinsically organized into dynamic, anticorrelated functional networks. Proc. Natl. Acad. Sci. U.S.A 102, 9673-9678.

Friston, K.J., Fletcher, P., Josephs, O., Holmes, A., Rugg, M.D., Turner, R., 1998. Eventrelated fMRl: characterizing differential responses. Neuroimage 7, 30-40.

Genovese, C.R., Lazar, N.A., Nichols, T., 2002. Thresholding of statistical maps in functional neuroimaging using the false discovery rate. Neuroimage 15 , $870-878$.

Grady, C., 2012. The cognitive neuroscience of ageing. Nat. Rev. Neurosci. 13, 491-505.

Grady. C..., Protzner, A.B., Kovacevic, N., Strother, S.C., Afshin-Pour, B. Wojtowicz, M., Anderson, JA., Churchill, N., Mclntosh, A.R., 2010. A multivariate analysis of age-related differences in default mode and task-positive networks across multiple cognitive domains. Cereb. Cortex 20, 1432-1447.

Greicius, M.D., Supekar, K., Menon, V., Dougherty, R.F., 2009. Resting-state functional connectivity reflects structural connectivity in the default mode network. Cereb. Cortex 19, 72-78.

He, Y., Wang, L., Zang, Y., Tian, L, Zhang, X., Li, K., Jiang, T., 2007. Regional coherence changes in the early stages of Alzheimer's disease: a combined structural and resting-state functional MRI study. Neuroimage 35, 488-500.

Himberg. J., Hyvarinen, A., Esposito, F., 2004. Validating the independent components of neuroimaging time series via clustering and visualization. Neuroimage 22, 1214-1222.

Huijbers, W.. Pennartz, C.M., Cabeza, R., Daselaar, S.M. 2011. The hippocampus is coupled with the default network during memory retrieval but not during memory encoding. Plos One 6, e17463.

Huster, R.J., Debener, S., Eichele, T., Herrmann, C.S., 2012. Methods for simultaneous EEG-fMRI: an introductory review. J. Neurosci. 32, 6053-6060.

Hutton, C. Bork, A. Josephs, O. Deichmann, R., Ashburner, J., Turner, R., 2002. Image distortion correction in fMRI: a quantitative evaluation. Neuroimage 16,217-240.

lannetti, G.D., Niazy. R.K., Wise, R.G., Jezzard, P., Brooks, J.C., Zambreanu, L., Vennart, W., Matthews, P.M. Tracey, 1., 2005. Simultaneous recording of laserevoked brain potentials and continuous, high-field functional magnetic resonance imaging in humans. Neuroimage 28, 708-719.

lannetti, G.D., Wise, R.G., 2007. BOL.D functional MRI in disease and pharmacological studies: room for improvement? Magn. Reson. Imaging 25, 978-988.

Jafri, M.J., Pearlson, G.D., Stevens, M., Calhoun, V.D., 2008. A method for functional network connectivity among spatially independent resting-state components in schizophrenia. Neuroimage 39, 1666-1681.

Jansen, M., White, T.P., Mullinger, K.J., Liddle, E.B., Gowland, PA., Francis, S.T., Bowtell, R., Liddle, P.F., 2012. Motion-related artefacts in EEG predict neuronally plausible patterns of activation in fMRI data. Neuroimage 59, 261-270.
Kannurpatti, S.S., Motes, M.A., Rypma, B., Biswal, B.B., 2010. Neural and vascular variability and the AMRI-BOLD response in normal aging. Magn. Reson. Imaging $28,466-476$.

Kelly, A.M., Uddin, LQ, Biswal, B.B., Castellanos, F.X. Milham, M.P., 2008. Competition between functional brain networks mediates behavioral variability. Neuroimage 39, 527-537.

Kelly, C., Biswal, B.B., Craddock, RC., Castellanos, FX., Milham, M.P., 2012. Characterizing variation in the functional connectome: promise and pitfalls. Trends. Cogn. Sci. 16, 181-188.

Klimesch, W., 1999. EEG alpha and theta oscillations reflect cognitive and memory performance: a review and analysis. Brain Res. Brain Res. Rev. 29, 169-195.

Langenecker, S.A., Nielson, KA., Rao, S.M., 2004. AMRI of healthy older adults during Stroop interference. Neuroimage 21, 192-200.

Laufs, H., Krakow, K., Sterzer, P., Eger, E. Beyerle, A., Salek-Haddadi, A. Kleinschmidt, A., 2003. Electroencephalographic signatures of attentional and cognitive default modes in spontaneous brain activity fluctuations at rest. Proc. Natl. Acad. Sci. U.SA 100, 11053-11058.

Li, Y.O., Adali, T., Calhoun, V.D., 2007. Estimating the number of independent components for functional magnetic resonance imaging data. Hum. Brain Mapp. $28,1251-1266$.

Lustig. C., Snyder, AZ. Bhakta, M. O'Brien, K.C., McAwoy, M., Raichle, M.E. Morris, J.C., Buckner, R.L., 2003. Functional deactivations: change with age and dementia of the Alzheimer type. Proc. Natl. Acad. Sci. U.S.A 100, 14504-14509.

Mantini, D., Perrucci, M.G. Del Gratta, C., Romani, G.L, Corbetta, M., 2007. Electrophysiological signatures of resting state networks in the human brain. Proc. Natl. Acad. Sci. U.SA 104, 13170-13175.

Michielse, S., Coupland, N., Camicioli, R., Carter, R., Seres, P., Sabino, J., Malykhin, N., 2010. Selective effects of aging on brain white matter microstructure: a diffusion tensor imaging tractography study. Neuroimage 52, 1190-1201.

Milham, M.P. Erickson, K.L., Banich, M.T., Kramer, A.F., Webb, A., Wszalek, T Cohen, N.J., 2002. Attentional control in the aging brain: insights from an fMR study of the stroop task. Brain Cogn. 49, 277-296.

Moosmann, M., Schonfelder, V.H., Specht, K., Scheeringa, R., Nordby, H., Hugdahl, K. 2009. Realignment parameter-informed artefact correction for simultaneous EEG-fMRI recordings. Neuroimage 45, 1144-1150.

Moretti, D.V., Babiloni, C., Binetti, G. Cassetta, E., Dal Forno, G. Ferreric, F., Ferri, R. Lanuzza, B., Miniussi, C. Nobili, F., Rodriguez, G.. Salinari, S., Rossini, P.M., 2004. Individual analysis of EEG frequency and band power in mild Alzheimer's disease. Clin. Neurophysiol. 115, 299-308.

Mullinger, K.J., Morgan, P.S., Bowtell, RW., 2008. Improved artifact correction for combined electroencephalography/functional MRI by means of synchronization and use of vectorcardiogram recordings. J. Magn. Reson. Imaging 27, 607-616.

Nelson, H.E., 1982. National Adult Reading Test. NFER-Nelson, Windsor, UK.

Niazy, R.K., Beckmann, C.F., lannetti, G.D., Brady. J.M., Smith, S.M., 2005. Removal of FMRI environment artifacts from EEG data using optimal basis sets. Neuroimage $28,720-737$.

Niazy, R.K., Xie, J., Miller, K., Beckmann, C.., Smith, S.M., 2011. Spectral characteristics of resting state networks. Prog. Brain Res. 193, 259-276.

Nolan, H., Whelan, R. Reilly, R.B., 2010. FASTER: Fully Automated Statistica Thresholding for EEG artifact Rejection. J. Neurosci. Methods 192, 152-162.

Nuwer, M., 1988. Quantitative EEG I: technique and problems of frequency analysis and topographic mapping. J. Clin. NeurophysioL. 5, 1-43.

Nyberg, L., Salami, A., Andersson, M., Eriksson, J. Kalpouzos, G., Kauppi, K., Lind, J. Pudas, S., Persson, J. Nilsson, L.G., 2010. Longitudinal evidence for diminished frontal cortex function in aging. Proc. Natl. Acad. Sci. U.S.A 107, 22682-22686.

O'Connell, R.G., Balsters, J.H., Kilcullen, S.M., Campbell, W., Bokde, A.W., Lai, R., Upton, N., Robertson, L.H., 2012. A simultaneous ERP/IMRI investigation of the P300 aging effect. Neurobiol. Aging 33, 2448-2461.

Power, J.D., Barnes, K.A., Snyder, AZ., Schlaggar, B.L., Petersen, S.E, 2012. Spurious but systematic correlations in functional connectivity MRI networks arise from subject motion. Neuroimage 59, 2142-2154.

Rajah, M.N., D'Esposito, M., 2005. Region-specific changes in prefrontal function with age: a review of PET and AMRI studies on working and episodic memory. Brain 128, 1964-1983.

Raz, N., Lindenberger, U., Rodrigue, K.M., Kennedy, K.M., Head, D., Williamson, A. Dahle, C. Gerstorf, D_. Acker, J.D., 2005. Regional brain changes in aging healthy adults: general trends, individual differences and modifiers. Cereb. Cortex 15 , $1676-1689$.

Richard Clark, C., Veltmeyer, M.D., Hamilton, R.J., Simms, E., Paul, R., Hermens, D., Gordon, E., 2004. Spontaneous alpha peak frequency predicts working memory performance across the age span. Int. J. Psychophysiol. 53, 1-9.

Sadaghiani, S. Scheeringa, R., Lehongre, K., Morillon, B. Giraud, A... Kleinschmidt, A., 2010. Intrinsic connectivity networks, alpha oscillations, and tonic alertness: a simultaneous electroencephalography/functional magnetic resonance imaging study. J. Neurosci. 30, 10243-10250.

Salami, A., Eriksson, J. Nyberg. L., 2012. Opposing effects of aging on large-scale brain systems for memory encoding and cognitive control. J. Neurosci. 32 , 10749-10757.

Salvador, R., Martinez, A., Pomarol-Clotet, E., Gomar, J. Vila, F., Sarro, S. Capdevila, A., Bullmore, E., 2008. A simple view of the brain through a frequency-specific functional connectivity measure. Neuroimage 39, 279-289.

Scheeringa, R., Fries, P., Petersson, K.M., Oostenveld, R., Grothe, 1., Norris, D.G. Hagoort, P., Bastiaansen, M.C, 2011. Neuronal dynamics underlying high- and low-frequency EEG oscillations contribute independently to the human BOLD signal. Neuron 69, 572-583. 
Schmahmann, J.D., Pandya, D.N., 2006. Fiber Pathways of the Brain. Oxford Universtiy Press, New York.

Smith, S.M., Fox, P.T., Miller, K.L., Glahn, D.C., Fox, P.M., Mackay, C.E, Filippini, N., Watkins, K.E. Toro, R., Laird, A.R. Beckmann, C.F., 2009. Correspondence of the brain's functional architecture during activation and rest. Proc. Natl. Acad. Sci. U.SA 106, 13040-13045.

Spreng, R.N., Wojtowicz, M., Grady, C.I., 2010. Reliable differences in brain activity between young and old adults: a quantitative meta-analysis across multiple

cognitive domains. Neurosci. Biobehav. Rev. 34, $1178-1194$.
Teipel, S.J., Bokde, A.L., Meindl, T., Amaro Jr., E., Soldner, J., Reiser, M.F., Herpertz, S.C., Moller, H.J., Hampel, H., 2010. White matter microstructure underlying default mode network connectivity in the human brain. Neuroimage 49, 2021-2032.

Teipel, S.J., Born, C., Ewers, M., Bokde, A.l., Reiser, M.F., Moller, H.J., Hampel, H., 2007. Multivariate deformation-based analysis of brain atrophy to predict Alzheimer's disease in mild cognitive impairment. Neuroimage 38, 13-24.

Van Dijk, K.R., Sabuncu, M.R. Buckner, R.L...2012. The influence of head motion on intrinsic functional connectivity MRL. Neuroimage 59, 431-438.

Verstynen, T.D., Deshpande, V., 2011. Using pulse oximetry to account for high and low frequency physiological artifacts in the BOLD signal. Neuroimage 55, 1633-1644.
Vincent, J.., Snyder, A.Z., Fox, M.D., Shannon, B.J., Andrews, J.R., Raichle, M.E. Buckner, R.L, 2006. Coherent spontaneous activity identifies a hippocampalparietal memory network. J. Neurophysiol. 96, 3517-3531.

Weschler, D., 1998. WMS-Illuk Administration and Scoring Manual, third ed. The Psychological Corporation Limited, New York.

Wise, R.G., Ide, K. Poulin, M.J. Tracey, 1. 2004. Resting fluctuations in arterial carbon dioxide induce significant low frequency variations in BOL.D signal. Neuroimage $21,1652-1664$

Ystad, M., Hodneland, E., Adolfsdottir, S., Haasz, J., Lundervold, A.J., Eichele, T. Lundervold, A., 2011. Cortico-striatal connectivity and cognition in normal aging: a combined DTI and resting state fMRI study. Neuroimage 55, 24-31.

Zhang. D. Raichle, M.E., 2010. Disease and the brain's dark energy. Nature Rev. Neurol. 6, 15-28.

Zigmond, A.S., Sims, A.C., 1983. The effect of the use of the International Classification of Diseases 9th revision: upon hospital in-patient diagnosis. Br. J. Psychiatry 142, 409-413.

Zysset, S., Schroeter, M.L., Neumann, J., von Cramon, D.Y., 2007. Stroop interference, hemodynamic response and aging: an event-related fMRI study. Neurobiol. Aging 28, 937-946. 